\title{
Stability Prediction of Coal Mine Water Disasters Emergency Rescue System Based on Support Vector Machine Yan- liang Zhang ${ }^{1}$ and Yang Liu ${ }^{2}$
}

School of Management and Engineering, Zhengzhou University, China

Email: 1119883038@qq.com

Key words: rescue system; stability; support vector machine; particle swarm; predication

\begin{abstract}
In order to effectively improve the stability of the coal mine water disasters emergency rescue system, the establishment of the index system to measure the stability standing on the view of mid-control and dynamic analysis, which includes six elements: personnel quality factor、capital factor、management factor 、 information elements machine elements and geological environment. As well, aiming at the characteristic of complex and data acquisition difficulty of coal miner rescue system, proposed a forecasting model of the stability of the coal mine water disasters emergency rescue system based on improved particle swarm optimization and support vector machine (IPSO-SVM). Finally, test the validity of the model through the case experiment.

Coal mine water disaster is one of the major disasters of the safe and efficient development of coal resources ${ }^{[1]}$.From a macro point of view, the coal mine water disaster in our country is on the rise; For coal mining enterprises, once the coal mine was flooded by sudden water, ore party are often helpless for water disasters, often delay the best time to rescue, as a result, the coal mine suffered serious economic losses, the miners and their families suffered a great disaster and pain. Therefore, the study of coal mine water disaster emergency rescue system is particularly important. Currently, the study mainly focused on the emergency rescue capability evaluation ${ }^{[2]}$ and rescue logistics management ${ }^{[3]}$, rarely systematically focused on the coal mine water disaster rescue system. In view of the characteristics of the coal mine water disaster rescue system that the system is complicated, the data acquisition is difficult, we propose a forecasting model of the stability of the coal mine water disasters emergency rescue system based on improved particle swarm optimization and support vector machine.
\end{abstract}

\section{The index system of coal mine water disaster rescue organization system stability}

Coal mine water disaster rescue is a complex dynamic system involving human- machine environment, engineering technology, and other elements, in order to improve the reaction efficiency and the quality of crisis management of coal mine, we take management of the stability of coal mine water disaster rescue system to measure the stability of coal mine rescue organization system and to improve the organizational system and to lay a solid foundation for the rescue work. The coal mine water disaster rescue organization system is established according to the thought of system engineering. The system includes personnel qualityB1、capital elementsB2、 management factorsB3、 information factorsB4、machine elementsB5、 geographical environment factorsB6.B1 includes the using proportion talentsC1、 the overall educational level of employeesC2 and the ability of employees to continue ascensionC3; B2 includes the proportion of business incomeC4 and the proportion of government investment incomeC5; B3 includes the degree of management systemC6 、 popularization of advanced management experienceC7 and the application of information managementC8; B4 includes the flow rate of informationC9 and the update rate of informationC10; B5 includes the update and maintenance rate of system equipmentC11 v the perfect degree of the waterproof and drainage systemC12 and the advanced level of relief means and technologyC13; B6 includes the ratio of special equipment and personnelC14 v the detection ability of geological environment C15 and the perfect degree of the hydrogeology dataC16. 


\section{The establishment of prediction model of coal mine water disaster rescue organization system stability}

There is a vague, non-linear relationship between the mine water disaster rescue organization system stability and affecting factors, the support vector machine with strict theoretical basis has a distinct advantage to solve the problem of nonlinear, high dimension and small sample: less data sample demand, high accuracy and strong adaptability, effective way to avoid the curse of dimensionality, etc. Therefore, the support vector machine water is introduced into the coal mine disaster relief organization system stability prediction, while selecting SVM kernel function and parameter as a breakthrough for in-depth study.

2.1 Basic learning method of support vector machines (SVM)

Vapnik [4] proposed the concept of kernel functions about SVM nonlinear classification surface problems, the basic idea is: a pre-determined by non-linear mapping of the input vector $\mathrm{x}$ is mapped to a high-dimensional space, then this high-dimensional space constructed optimal classification surface.

Set of training data set $\left\{\left(x_{i}, y_{i}\right), i=1,2, \cdots \cdots n\right\}$ for the size of $\mathrm{n}, y \subset\{+1,-1\}$ can be transformed into quadratic optimization problem:

The constraint conditions: $y_{i}\left(w \cdot x_{i}+b\right) \geq 1 \quad i=1,2, \cdots n$

Next, seek the minimum value. The solution to this optimization problem is a saddle point of the following Lagrange function:

$$
L(\mathrm{w}, b, a)=\frac{1}{2}\|w\|^{2}-\sum_{i=1}^{n} a_{i}\left[y_{i}\left(w \cdot x_{i}+b\right)-1\right]
$$

While $a_{i}$ is non-negative Lagrange multipliers. Equation (2) is a convex quadratic programming problem, so there is a globally unique optimal solution. Due to the complexity of the calculation, generally do not directly solve, but according to Lagrange duality theory equation (2), it is converted to the dual problem, while using kernel functions satisfied Mercer condition instead of the vector plot point calculation, namely

$$
\left\{\begin{array}{l}
\max Q(a)=\sum_{i=1}^{n} a_{i}-\frac{1}{2} \sum_{i=1}^{n} \sum_{i=1}^{n} a_{i} a_{j} y_{i} y_{j} K\left(x_{i}, x_{j}\right) \\
\text { s.t. }\left\{\begin{array}{l}
\sum_{i=1}^{n} a_{i} y_{i}=0 \\
0 \leq a_{i} \leq c
\end{array} \quad \dot{i}=1,2, \cdots, n\right.
\end{array}\right.
$$

If $a_{i}^{*}$ is the formula (3) optimal solution, then

$$
\mathrm{w}^{*}=\sum_{i=1}^{n} a_{i}^{*} y_{i} x_{i}
$$

Ultimately the optimal classification function is:

$$
\mathrm{f}(\mathrm{x})=\operatorname{sgn}\left(\sum_{i=1}^{n} a_{i}^{*} y_{i} K\left(x_{i}, x_{j}\right)+b^{*}\right)
$$

The classification of $\mathrm{x}$ can be determined according to the value of $f(x)$ with 1 or -1 .

2.2The selection of kernel function

Kernel function is in the central position in SVM data processing, which plays a crucial role. RBF kernel function can not only adapt to the complexity of the model, and need only a selection of variance, and has the very good fitting in the high-dimensional space, so choose RBF as the kernel

$$
K\left(x, x_{i}\right)=\exp \left(\frac{-\left\|x-x_{i}\right\|^{2}}{2 \delta^{2}}\right)
$$
function of support vector machines (SVM), RBF kernel function [5] is as follows: 
2.3Parameters optimization based on improved particle swarm algorithm

(1) The principle of PSO algorithm

Particle swarm optimization was a population of intelligent optimization algorithm originally founded in 1995 by Dr Eberhart and Dr Keenedy [5] in the United States. The algorithm is an algorithm for the simulation of bionic bird foraging, simple operation principle as follows: Assuming the position of the particle swarm in the particle of $\mathrm{i}$ in D-dimensional space is represented as an n-dimensional vector $x_{i}=\left(x_{i 1}, x_{i 2}, \cdots, x_{i j}, \cdots, x_{i D}\right)$, speed $^{V_{i}}=\left(v_{i 1}, V_{i 2}, \cdots, v_{i j}, \cdots, V_{i D}\right)$ determines the displacement of particles in search space of a single iteration. Current particle swarm optimal individual particles is $p_{i}=\left(p_{i 1}, \mathrm{p}_{i 2}, \cdots, p_{i j}, \cdots, p_{i D}\right)$, the global optimal particle is expressed ${ }_{\text {as }} \mathrm{g}=\left(\mathrm{g}_{1}, \mathrm{~g}_{2}, \cdots, \mathrm{g}_{\mathrm{j}}, \cdots \mathrm{g}_{\mathrm{D}}\right)$, each particle according to the formula (7) update their position and velocity until the iteration termination condition is satisfied.

$$
\left\{\begin{array}{l}
v_{i j}=w V_{i j}+c_{1} r_{1}\left(p_{i j}-x_{i j}\right)+c_{2} r_{2}\left(g_{j}-x_{i j}\right) \\
x_{i j}=x_{i j}+v_{i j}
\end{array}\right.
$$

The inertia weight $\mathrm{w}$, learning weight $c_{1}$ and weight $c_{2}$ are non-negative, and $r_{1}$ 和 $r_{2}$ is the random number of change within the scope of $[0,1]$, the speed $\mathrm{V}_{\mathrm{i}}$ is set between $\mathrm{V}_{\min }$ and $V_{\max }$ to prevent particle velocity out of control.

(2)The improvement of PSO algorithm

PSO has some advantages such as less parameters, simple principle, easy to implement, exist poor local search ability and search accuracy is not enough higher. In order to solve the above problems from inertia weight $\mathrm{w}$ and learning weight $\mathrm{c}$, the inertia weight $\mathrm{w}$ plays a key role for the convergence of PSO, using linear decreasing inertia weight adjustment can reduce $\mathrm{w}$ in the process of iteration step by step, which is helpful to enhance the search intensity, the adjusting formula is shown in formula (8); Learning weight and inertia weight, earning weight plays a role similar to inertia weight by adjusting the weights to improve PSO's convergence, the adjustment formula is shown in formula (9).

$$
\begin{aligned}
& W=W_{\text {max }}-\left(W_{\text {max }}-W_{\text {min }}\right) \cdot t / T \\
& c_{1}=c_{2}=c_{\text {max }}-\left(c_{\text {max }}-c_{\text {min }}\right) \cdot t / T
\end{aligned}
$$

While $\mathrm{t}$ is the current iteration number, $\mathrm{T}$ is the maximum number of iterations.

(3)Parameters optimization based on IPSO

By above knowable chosen RBF function is selected as the kernel function of support vector machines (SVM), so the model has two parameters: penalty factor $C$ and variance of RBF function. The classification accuracy rate through parameter optimization mechanism CV is selected as a function of PSO algorithm, the higher accuracy, and the stronger adaptability. Specific approach is: Calculating the mean classification accuracy of the $\mathrm{K}$ model through $\mathrm{K}-\mathrm{CV}$ algorithm and using it as an application degree function of particle swarm algorithm, denoted by $F(C, \delta)=a_{K-C V}$, calculate and find the fitness of the largest particles. The process through making use of IPSO to optimize parameters of the support vector machine is as follows:

(1)Set the searching scope of the SVM parameter and initialize IPSO algorithm parameters.

(2)Initialize velocity and position of the particle.

(3)Determine the applicable function F and train SVM.

(4)Calculate the fit value of the particle, initialize the $\mathrm{p}$ and $\mathrm{g}$.

(5)According to the formula (8) and (9) to update the particle's speed and position, update the p 
and $g$.

(6)Seeing if it reaches the maximum number of iterations $\mathrm{T}$, the answer is no, then repeat the step (5); the answer is yes, then continue the next step.

(7)Output the optimal combined penalty factor and parameters of kernel function.

2.4 The mine water disaster rescue organization system stability prediction model framework based on IPSO-SVM

The predictive model of coal mine water disaster rescue organization system stability is mainly divided into three parts: sample processing, parameter optimization and model training. Specific forecasting process is as follows:

(1) Processing the original sample, including the classification of steady-state operation and data standardization, played the role of de-noising and simplified.

(2) The initial position and velocity of the particle, a reasonable choice of initial parameters, define the use of the function, and the adjustment of the inertia weight $\mathrm{w}$ and learning the weight $\mathrm{c}$, through the iterative operation to find out the optimal value.

(3) The optimal combination of the parameters C is concluded through (1) and (2), then put it into the support vector machine for training, eventually come to an ideal model to predict stability.

(4) Testing samples through using the prediction mode obtained from (3) to verify the validity of the model.

\section{The empirical analysis}

3.1 steady-state classification

According to the commonly used industrial rescue steady state classification standard, the stability condition index set of the coal mine water disaster rescue organization system is $\mathrm{p}=\{$ not very stable; unstable; general; stable; very stable $\}$, and the corresponding quantitative vector is $U=\{1 ; 2 ; 3 ; 4 ; 5\}$, set the stability result sets of coal mine water disaster relief organization system as $\mathrm{P}=\{$ unstable; stable $\}$, and the corresponding quantitative vector is $U=\{0 ; 1\}$, According to the mine rescue regulations, field survey and expert advice, categorizing the stability index of the subsystems of the division, as shown in table 1.

Table 1 steady state classification of coal mine rescue system

\begin{tabular}{|c|c|c|c|c|c|}
\hline \multirow[t]{2}{*}{ indicators } & \multicolumn{5}{|c|}{ steady state classification } \\
\hline & 1 & 2 & 3 & 4 & 5 \\
\hline $\mathrm{C}_{1}$ & $\leq 0.45$ & $0.45 \sim 0.60$ & $0.60 \sim 0.70$ & $0.70 \sim 0.80$ & $\geq 0.80$ \\
\hline $\mathrm{C}_{2}$ & $\leq 45$ & $45 \sim 55$ & $55 \sim 65$ & $65 \sim 75$ & $\geq 75$ \\
\hline $\mathrm{C}_{3}$ & $\leq 50$ & $50 \sim 60$ & $60 \sim 70$ & $70 \sim 80$ & $\geq 80$ \\
\hline $\mathrm{C}_{4}$ & $\leq 0.1$ & $0.1 \sim 0.2$ & $0.2 \sim 0.3$ & $0.3 \sim 0.4$ & $\geq 0.4$ \\
\hline $\mathrm{C}_{5}$ & $\leq 0.2$ & $0.2 \sim 0.3$ & $0.3 \sim 0.4$ & $0.4 \sim 0.5$ & $\geq 0.5$ \\
\hline $\mathrm{C}_{6}$ & $\leq 65$ & $65 \sim 75$ & $75 \sim 85$ & $85 \sim 95$ & $\geq 95$ \\
\hline $\mathrm{C}_{7}$ & $\leq 65$ & $65 \sim 75$ & $75 \sim 85$ & $85 \sim 95$ & $\geq 95$ \\
\hline $\mathrm{C}_{8}$ & $\leq 65$ & $65 \sim 75$ & $75 \sim 85$ & $85 \sim 95$ & $\geq 95$ \\
\hline $\mathrm{C}_{9}$ & $\leq 10$ & $10 \sim 20$ & $20 \sim 30$ & $30 \sim 40$ & $\geq 40$ \\
\hline $\mathrm{C}_{10}$ & $\leq 15$ & $15 \sim 25$ & $25 \sim 35$ & $35 \sim 45$ & $\geq 45$ \\
\hline $\mathrm{C}_{11}$ & $\leq 30$ & $30 \sim 40$ & $40 \sim 50$ & $50 \sim 60$ & $\geq 60$ \\
\hline $\mathrm{C}_{12}$ & $\leq 65$ & $65 \sim 75$ & $75 \sim 85$ & $85 \sim 95$ & $\geq 95$ \\
\hline $\mathrm{C}_{13}$ & $\leq 65$ & $65 \sim 75$ & $75 \sim 85$ & $85 \sim 95$ & $\geq 95$ \\
\hline $\mathrm{C}_{14}$ & $\leq 0.3$ & $0.3 \sim 0.4$ & $0.4 \sim 0.5$ & $0.5 \sim 0.6$ & $\geq 0.6$ \\
\hline $\mathrm{C}_{15}$ & $\leq 65$ & $65 \sim 75$ & $75 \sim 85$ & $85 \sim 95$ & $\geq 95$ \\
\hline $\mathrm{C}_{16}$ & $\leq 65$ & $65 \sim 75$ & 75 85 & $85 \sim 95$ & $\geq 95$ \\
\hline
\end{tabular}

3.2 Obtaining and processing of the sample data 
According to the index system of coal mine water disaster rescue combination system stability, on the basis of investigation of the $\mathrm{X}$ coal mine, select 16 groups of the mine rescue system related data over a period of time through the original data record. In order to improve the classification accuracy of steady state, to eliminate different variables, to eliminate the influence of different level on the predicted results, according to the division of steady state categories in table 1, de-noising the original data and simplify the processing, as shown in table 2.

\begin{tabular}{|c|c|c|c|c|c|c|c|c|c|c|c|c|c|c|c|c|}
\hline sample indi & 1 & 2 & 3 & 4 & 5 & 6 & 7 & 8 & 9 & 10 & 11 & 12 & 13 & 14 & 15 & 16 \\
\hline $\mathrm{C}_{1}$ & 5 & 2 & 4 & 5 & 4 & 1 & 5 & 5 & 5 & 1 & 2 & 4 & 1 & 4 & 2 & 5 \\
\hline $\mathrm{C}_{2}$ & 5 & 1 & 3 & 4 & 5 & 2 & 4 & 5 & 4 & 1 & 2 & 3 & 1 & 4 & 2 & 5 \\
\hline $\mathrm{C}_{3}$ & 5 & 1 & 5 & 5 & 5 & 1 & 4 & 4 & 4 & 2 & 3 & 5 & 1 & 5 & 1 & 4 \\
\hline $\mathrm{C}_{4}$ & 4 & 2 & 2 & 4 & 5 & 2 & 5 & 5 & 5 & 3 & 3 & 5 & 1 & 4 & 2 & 5 \\
\hline $\mathrm{C}_{5}$ & 5 & 1 & 5 & 4 & 5 & 1 & 5 & 4 & 4 & 1 & 2 & 5 & 1 & 4 & 2 & 4 \\
\hline $\mathrm{C}_{6}$ & 5 & 2 & 5 & 4 & 5 & 4 & 4 & 5 & 4 & 3 & 1 & 5 & 1 & 4 & 2 & 4 \\
\hline $\mathrm{C}_{7}$ & 5 & 2 & 5 & 5 & 4 & 2 & 3 & 5 & 5 & 1 & 2 & 4 & 1 & 5 & 1 & 4 \\
\hline $\mathrm{C}_{8}$ & 5 & 1 & 3 & 3 & 4 & 1 & 5 & 5 & 4 & 2 & 1 & 4 & 2 & 3 & 1 & 5 \\
\hline $\mathrm{C}_{9}$ & 4 & 2 & 5 & 5 & 4 & 1 & 4 & 4 & 4 & 3 & 3 & 5 & 1 & 5 & 2 & 5 \\
\hline $\mathrm{C}_{10}$ & 5 & 2 & 4 & 3 & 4 & 3 & 4 & 4 & 3 & 1 & 3 & 4 & 1 & 4 & 2 & 4 \\
\hline $\mathrm{C}_{11}$ & 5 & 3 & 5 & 3 & 5 & 2 & 3 & 5 & 4 & 3 & 2 & 3 & 1 & 5 & 2 & 4 \\
\hline $\mathrm{C}_{12}$ & 5 & 1 & 4 & 5 & 4 & 1 & 5 & 5 & 5 & 1 & 2 & 4 & 1 & 4 & 2 & 5 \\
\hline $\mathrm{C}_{13}$ & 4 & 1 & 5 & 4 & 5 & 2 & 4 & 4 & 5 & 3 & 2 & 5 & 1 & 5 & 2 & 3 \\
\hline $\mathrm{C}_{14}$ & 5 & 2 & 1 & 2 & 5 & 1 & 1 & 5 & 2 & 2 & 1 & 5 & 2 & 4 & 1 & 5 \\
\hline $\mathrm{C}_{15}$ & 5 & 1 & 2 & 5 & 4 & 1 & 5 & 4 & 5 & 2 & 1 & 3 & 2 & 5 & 3 & 4 \\
\hline $\mathrm{C}_{16}$ & 5 & 1 & 5 & 4 & 3 & 2 & 5 & 4 & 5 & 1 & 1 & 4 & 1 & 4 & 1 & 5 \\
\hline $\begin{array}{c}\text { stable } \\
\text { category }\end{array}$ & 1 & 0 & 1 & 1 & 1 & 0 & 1 & 1 & 1 & 0 & 0 & 1 & 0 & 1 & 0 & 1 \\
\hline
\end{tabular}

\subsection{The operation of the experiment}

In order to verify the validity of the prediction model, in Matlab7.0 [6] simulation environment, using Libsvm-3.1toolki, as particle swarm optimization function applies simulation experiments. Selecting 1to12 samples of table 3 for the training set, 13 to16 samples for the test set. Initial experimental parameter settings are as follows: the value of particle dimension (D) is 2; the value of the maximum learning weight is 1.7 ; the value of the minimum learning weight is 0.9 ; the value of the maximum inertia weight is 0.9 ; the value of the minimum inertia weight is 0.4 ; the maximum number of iterations $(\mathrm{T})$ is 200; the maximum speed of the particle is 1.2; penalty factor $\mathrm{C}$ of $\mathrm{SVM}$ is $[0.1,100]$ and kernel Parameter is $[0.1,50]$.

After the adjustment of the inertia weight and learning weight, IPSO is obviously enhanced on the precision and convergence speed, IPSO compared with PSO the convergence curve is more stable, verifying the effectiveness of the SVM parameters based on IPSO optimization. Summarizing the results of the parameter optimization can be drawn IPSO has a great advantage in terms of error rate and the running time, it is possible to avoid premature local optimum, as shown in Table 5.

Table 5 comparison of parameter optimization results

\begin{tabular}{ccccc}
\hline algorithm & parameter C & parameter $\delta$ & time T & the error rate \\
\hline IPSO & 8.181 & 2.350 & $0.98 \mathrm{~s}$ & $8 \%$ \\
PSO & 7.781 & 1.910 & $1.52 \mathrm{~s}$ & $16 \%$ \\
\hline
\end{tabular}

\section{4 model test results}

After studying and fitting all the 12 training sample set, respectively using IPSO - SVM and PSO 
- SVM model to forecast the four test sample set, predicted results are as shown in table 6.

\begin{tabular}{cccccccc}
\multicolumn{7}{c}{ Table 6 test results of IPSO - SVM and PSO - SVM model } & \\
\hline model & $\begin{array}{c}\text { samples } \\
\text { serial number }\end{array}$ & 13 & 14 & 15 & 16 & accuracy & parameter $(\mathrm{C}, \delta)$ \\
\hline IPSO-SVM & the actual value & 0 & 1 & 0 & 1 & $100 \%$ & $(8.818,2.350)$ \\
& predictive value & 0 & 1 & 0 & 1 & & \\
PSO-SVM & the actual value & 0 & 1 & 0 & 1 & $75 \%$ & $(7.781,1.910)$ \\
& predictive value & 0 & $(0)$ & 0 & 1 & &
\end{tabular}

As can be seen from Table 6, the predicted results of PSO - SVM model have appeared a sample classification error, the accuracy rate reached 75\%, IPSO-SVM prediction accuracy rate reached 100 percent, which can not only show that the SVM has a unique advantage in solving the nonlinear binary classification, but also more proof of the validity of the mine water disaster rescue organization system stability prediction model based on IPSO-SVM.

\section{Conclusion}

This paper can get the following conclusions through empirical analysis:

(1)This article follows the basic principles of systematic, comprehensive, dynamic, and combining with the status quo and expert advice to establish the index system of coal mine water disaster relief combination system stability.

(2)Coal mine water disaster relief organization system stability prediction model was proposed based on IPSO-SVM, for coal mine water disaster relief system is complicated and the data is difficult to get. The SVM has a unique advantage in solving nonlinear binary classification problem; Carrying on the linear adjustment for the study weight and inertia weight of particle swarm algorithm, effectively improve the precision and convergence speed of traditional particle swarm optimization; Finally, combined the improved particle swarm optimization with support vector machine (SVM), and the model parameters are optimized. And the effectiveness of the proposed model has been proved by the example.

\section{References}

[1]Wang Jin-feng, Yang Li-feng, Feng Li-jie. Response optimization for coal mine water disasters based on rough set [J]. Industrial Engineering Journal, 2013, 16(5):1-5.

[2]Yue Ning-fang. Establish assessing index system for the emergency rescue of serious disaster and accidents in the coal mining enterprises [J]. Coal Technology, 2009, 28(2):2-5.

[3]Li Jia-bin. Study on structural model of coal emergency logistics management [J].Logistics Engineering Management, 2014, 36(4):9-11.

[4] V.Vapnil. An Overview of Statistical Learning Theory [J].IEEE Transaction on Neural Networks Vol.10.No.5pp.988-999, 1999

[5]Eberhart R, Kennedy J. A new optimizer using particle swarm theory[C], Proc.6 Int. Symposium on Micro Machine and Human Science, 1995, pp. 39-43.

[6]Guo Xiao-hui, Ma Xiao-ping. Support vector machines toolbox in MATLAB environment [J].Computer Applications and Software, 2007, 24(12):57-59. 